\title{
Front Matter: Volume 6923
}

, "Front Matter: Volume 6923," Proc. SPIE 6923, Advances in Resist Materials and Processing Technology XXV, 692301 (17 April 2008); doi:

10.1117/12.798687

SPIE. Event: SPIE Advanced Lithography, 2008, San Jose, California, United States 


\title{
PROCEEDINGS OF SPIE
}

\section{Advances in Resist Materials and Processing Technology XXV}

\author{
Clifford L. Henderson \\ Editor
}

25-27 February 2008

San Jose, California, USA

Sponsored by

SPIE

Cooperating Organization

SEMATECH (USA)

Published by

SPIE 
The papers included in this volume were part of the technical conference cited on the cover and title page. Papers were selected and subject to review by the editors and conference program committee. Some conference presentations may not be available for publication. The papers published in these proceedings reflect the work and thoughts of the authors and are published herein as submitted. The publisher is not responsible for the validity of the information or for any outcomes resulting from reliance thereon.

Please use the following format to cite material from this book:

Author(s), "Title of Paper," in Advances in Resist Materials and Processing Technology XXV, edited by Clifford L. Henderson, Proceedings of SPIE Vol. 6923 (SPIE, Bellingham, WA, 2008) Article CID Number.

ISSN 0277-786X

ISBN 9780819471086

Published by

SPIE

P.O. Box 10, Bellingham, Washington 98227-0010 USA

Telephone +1 3606763290 (Pacific Time) · Fax +1 3606471445

SPIE.org

Copyright (C) 2008, Society of Photo-Optical Instrumentation Engineers

Copying of material in this book for internal or personal use, or for the internal or personal use of specific clients, beyond the fair use provisions granted by the U.S. Copyright Law is authorized by SPIE subject to payment of copying fees. The Transactional Reporting Service base fee for this volume is $\$ 18.00$ per article (or portion thereof), which should be paid directly to the Copyright Clearance Center (CCC), 222 Rosewood Drive, Danvers, MA 01923. Payment may also be made electronically through CCC Online at copyright.com. Other copying for republication, resale, advertising or promotion, or any form of systematic or multiple reproduction of any material in this book is prohibited except with permission in writing from the publisher. The CCC fee code is 0277-786X/08/\$18.00.

Printed in the United States of America.

Publication of record for individual papers is online in the SPIE Digital Library.

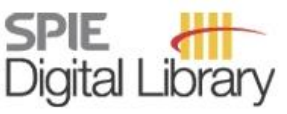

SPIEDigitalLibrary.org

Paper Numbering: Proceedings of SPIE follow an e-First publication model, with papers published first online and then in print and on CD-ROM. Papers are published as they are submitted and meet publication criteria. A unique, consistent, permanent citation identifier (CID) number is assigned to each article at the time of the first publication. Utilization of CIDs allows articles to be fully citable as soon they are published online, and connects the same identifier to all online, print, and electronic versions of the publication. SPIE uses a six-digit CID article numbering system in which:

- The first four digits correspond to the SPIE volume number.

- The last two digits indicate publication order within the volume using a Base 36 numbering

system employing both numerals and letters. These two-number sets start with $00,01,02,03,04,05$, $06,07,08,09,0 A, 0 B \ldots .0 Z$, followed by 10-1Z, 20-2Z, etc.

The CID number appears on each page of the manuscript. The complete citation is used on the first page, and an abbreviated version on subsequent pages. Numbers in the index correspond to the last two digits of the six-digit CID number. 


\title{
Contents
}

\section{Part One}

\author{
xvii Symposium Committees \\ xix Conference Committee
}

\section{SESSION 1 KEYNOTE SESSION}

692302 Rise of chemical amplification resists from laboratory curiosity to paradigm enabling Moore's law [6923-01]

H. Ito, IBM Almaden Research Ctr. (USA)

\section{SESSION 2 MATERIALS AND PROCESSES FOR IMMERSION LITHOGRAPHY I}

692304 The limitations of high-index resists for 193-nm hyper-NA lithography [6923-03]

G. McIntyre, IBM Advanced Lithography Research (USA); D. Sanders, R. Sooriyakumaran, H. Truong, R. Allen, IBM Almaden Research Ctr. (USA)

692305 High-index resist for 193-nm immersion lithography [6923-04]

K. Matsumoto, Tokyo Institute of Technology (Japan); E. Costner, I. Nishimura, The Univ. of Texas at Austin (USA); M. Ueda, Tokyo Institute of Technology (Japan); C. G. Willson, The Univ. of Texas at Austin (USA)

692306 Development of an operational high refractive index resist for 193nm immersion lithography [6923-05]

P. A. Zimmerman, J. Byers, E. Piscani, B. Rice, SEMATECH (USA); C. K. Ober, E. P. Giannelis, R. Rodriguez, D. Wang, Cornell Univ. (USA); A. Whittaker, I. Blakey, L. Chen, B. Dargaville,

H. Liu, Univ. of Queensland (Australia)

692307 Non-topcoat resist design for immersion process at 32-nm node [6923-06]

S. Wu, A. Tseng, B. Lin, C. C. Yu, B.-J. Lu, W.-S. Liao, United Microelectronics Corp. (Taiwan, China); D. Wang, V. Vohra, C. B. XU, S. Caporale, G. Barclay, Rohm and Haas Electronic Materials (USA)

\section{SESSION 3 MATERIALS AND PROCESSES FOR IMMERSION LITHOGRAPHY II}

692309 Self-segregating materials for immersion lithography [6923-08]

D. P. Sanders, L. K. Sundberg, P. J. Brock, H. Ito, H. D. Truong, R. D. Allen, IBM Almaden Research Ctr. (USA); G. R. McIntyre, IBM Advanced Lithography Research (USA);

D. L. Goldfarb, IBM Thomas J. Watson Research Ctr. (USA) 
6923 0A Development and evaluation of $193 \mathrm{~nm}$ immersion generation-three fluid candidates [6923-09]

P. A. Zimmerman, J. Byers, B. Rice, SEMATECH (USA); C. K. Ober, E. P. Giannelis,

R. Rodriguez, D. Wang, Cornell Univ. (USA); N. O'Connor, X. Lei, N. J. Turro, Columbia Univ. (USA); V. Liberman, S. Palmacci, M. Rothschild, MIT Lincoln Lab. (USA); N. Lafferty,

B. W. Smith, Rochester Institute of Technology (USA)

6923 OB New high-index fluids: exploiting anomalous dispersion for immersion lithography [6923-10] E. A. Costner, The Univ. of Texas at Austin (USA); K. Matsumoto, The Univ. of Texas at Austin (USA) and Tokyo Institute of Technology (Japan); B. K. Long, J. C. Taylor, The Univ. of Texas at Austin (USA); W. Wojtczak, SACHEM, Inc. (USA); C. G. Willson, The Univ. of Texas at Austin (USA)

6923 OC A new class of low bake resists for 193-nm immersion lithography [6923-11] R. Sooriyakumaran, R. DiPietro, H. Truong, P. Brock, R. Allen, L. Bozano, IBM Almaden Research Ctr. (USA); I. Popova, W.-S. Huang, R. Chen, M. Khojasteh, P. R. Varanasi, IBM Systems and Technology Group (USA)

6923 OD Process development for high scan speed Arf immersion lithography [6923-12] N. Matsumura, N. Sugie, K. Goto, K. Fujiwara, Y. Yamaguchi, JSR Corp. (Japan); H. Tanizaki, K. Nakano, T. Fujiwara, Nikon Corp. (Japan); S. Wakamizu, H. Takeguchi, H. Arima, H. Kyoda, K. Yoshihara, J. Kitano, Tokyo Electron Kyushu Ltd. (Japan)

6923 OE Immersion resist process for 32-nm node logic devices [6923-13]

T. Ema, K. Sho, H. Yonemitsu, Y. Seino, H. Fujise, A. Yamada, S. Mimotogi, Y. Kitamura, S. Nagai, K. Fujii, T. Fukushima, T. Komukai, A. Nomachi, T. Azuma, S. Ito, Toshiba Corp. (Japan)

\section{SESSION 4 MATERIALS AND PROCESSES FOR DOUBLE PATTERNING/DOUBLE EXPOSURE}

6923 OF Development of materials and processes for double patterning toward 32-nm node 193-nm immersion lithography process [6923-14]

S. Tarutani, H. Tsubaki, S. Kanna, FUJIFILM Corp. (Japan)

6923 OG Resist freezing process for double exposure lithography [6923-16]

K.-J. R. Chen, W.-S. Huang, W.-K. Li, P. R. Varanasi, IBM Semiconductor Research and Development Ctr. (USA)

$6923 \mathrm{OH}$ Sub-40-nm half-pitch double patterning with resist freezing process [6923-17] M. Hori, T. Nagai, A. Nakamura, T. Abe, G. Wakamatsu, T. Kakizawa, Y. Anno, M. Sugiura, S. Kusumoto, Y. Yamaguchi, T. Shimokawa, JSR Corp. (Japan)

6923 Ol Rigorous physical modeling of a materials-based frequency doubling lithography process [6923-18]

S. A. Robertson, J. J. Biafore, T. Graves, M. D. Smith, KLA-Tencor (USA)

\section{SESSION 5 MOLECULAR RESISTS}

6923 0J Novel molecular resist based on an amorphous truxene derivative [6923-20]

S. Hattori, S. Saito, K. Asakawa, T. Koshiba, T. Nakasugi, Toshiba Corp. (Japan) 
6923 OK Single molecule chemically amplified resists based on ionic and non-ionic PAGs [6923-21] R. A. Lawson, C.-T. Lee, Georgia Institute of Technology (USA); W. Yueh, Intel Corp. (USA);

L. Tolbert, C. L. Henderson, Georgia Institute of Technology (USA)

$6923 \mathrm{OL}$ Surface roughness of molecular resist for EUV lithography [6923-22]

M. Toriumi, Semiconductor Leading Edge Technologies, Inc. (Japan) and Lab. for Interdisciplinary Science and Technology (Japan); K. Kaneyama, S. Kobayashi, T. Itani, Semiconductor Leading Edge Technologies, Inc. (Japan)

6923 OM Chemically amplified fullerene resists for e-beam lithography [6923-23]

J. Manyam, F. P. Gibbons, S. Diegoli, M. Manickam, J. A. Preece, R. E. Palmer,

A. P. G. Robinson, The Univ. of Birmingham (United Kingdom)

$6923 \mathrm{ON}$ Chemically amplified molecular resist based on fullerene derivative for nanolithography [6923-24]

H. Yamamoto, T. Kozawa, S. Tagawa, Osaka Univ. (Japan); T. Ando, K. Ohmori, M. Sato, J. Onodera, Tokyo Ohka Kogyo Co., Ltd. (Japan)

$692300 \quad$ New architectures for high resolution patterning [6923-25]

A. De Silva, N. Felix, D. Forman, J. Sha, C. K. Ober, Cornell Univ. (USA)

\section{SESSION 6 SIMULATION OF RESIST PROCESSES}

6923 OP Base quencher effects in chemically amplified resist at sub-30-nm fabrication [6923-26] T. Kozawa, S. Tagawa, Osaka Univ. (Japan); J. J. Santillan, M. Toriumi, T. Itani, Semiconductor Leading Edge Technologies, Inc. (Japan)

$69230 Q \quad$ Mesoscale simulation of molecular glass photoresists: effect of PAG loading and acid diffusion coefficient [6923-27]

R. A. Lawson, C.-T. Lee, Georgia Institute of Technology (USA); W. Yueh, Intel Corp. (USA);

L. Tolbert, C. L. Henderson, Georgia Institute of Technology (USA)

6923 OR A comprehensive resist model for the prediction of line-edge roughness material and process dependencies in optical lithography [6923-28]

T. Schnattinger, A. Erdmann, Fraunhofer Institute of Integrated Systems and Device Technology (Germany)

6923 OS Monte Carlo simulation on line edge roughness after development in chemically amplified resist of post-optical lithography [6923-29]

A. Saeki, T. Kozawa, S. Tagawa, Osaka Univ. (Japan); H. B. Cao, H. Deng, M. J. Leeson, Intel Corp. (USA)

6923 OT Extraction and identification of resist modeling parameters for EUV lithography [6923-30]

C. A. Fonseca, Tokyo Electron America, Inc. (USA); R. Gronheid, IMEC (Belgium);

S. A. Scheer, Tokyo Electron America, Inc. (USA) 
6923 OU Etching spin-on trilayer masks [6923-32]

D. J. Abdallah, S. Miyazaki, A. Hishida, A. Timko, D. McKenzie, D. Rahman, W. Kim, L. Pylneva, H. WU, R. Zhang, P.-H. LU, M. Neisser, R. Dammel, AZ Electronic Materials (USA) and AZ Electron Materials (Japan)

$6923 \mathrm{OV}$ Graded spin-on organic bottom antireflective coating for high NA lithography [6923-33] D. L. Goldfarb, S. D. Burns, L. Vyklicky, D. Pfeiffer, IBM Thomas J. Watson Research Ctr. (USA); A. Lisi, K. Petrillo, J. Arnold, IBM Systems \& Technology Group (USA); D. P. Sanders, IBM Almaden Research Ctr. (USA); A. Clancy, R. N. Lang, IBM Systems \& Technology Group (USA); R. D. Allen, IBM Almaden Research Ctr. (USA); D. R. Medeiros, IBM Systems \& Technology Group (USA); D. C. Owe-Yang, Shin-Etsu MicroSi, Inc. (USA); K. Noda, S. Tachibana, S. Shirai, Shin-Ełsu Chemical Co., Ltd. (Japan)

6923 OW A high-Si content middle layer for ArF trilayer patterning [6923-34] J. Kennedy, S. Xie, R. Katsanes, K. Flanigan, S. Mukhopadhyay, B. Wu, E. W. Rutter, Jr., Honeywell (USA); M. Slezak, JSR Micro, Inc. (USA)

6923 0X Anti-reflective coating for multipatterning lithography [6923-35]

D. J. Guerrero, S. Gibbons, J. Lowes, R. Mercado, Brewer Science, Inc. (USA)

6923 OY A modified bilayer resist approach for $\mathbf{4 5} \mathbf{~ n m}$ flash lithography [6923-36] B. Osborn, G. Quinto, C. Cheung, F. Wang, C. Gabriel, F. Cheung, F. Tsai, A. Minvielle, Spansion, Inc. (USA)

\section{SESSION 8 EUV RESISTS}

$69230 Z$ A high-throughput contact-hole resolution metric for photoresists: full-process sensitivity study [6923-37]

C. N. Anderson, University of California, Berkeley (USA); P. P. Naulleau, Lawrence Berkeley National Lab. (USA)

692310 Resist development to improve flare issue of EUV lithography [6923-38] M. Irie, T. Suzuki, T. Mimura, T. Iwai, Tokyo Ohka Kogyo Co., Ltd. (Japan)

692312 Synthesis and properties of new anionic photoacid generators bound polymer resists for e-beam and EUV lithography [6923-40]

M. Wang, The Univ. of North Carolina at Charlotte (USA); C.-T. Lee, C. L. Henderson, Georgia Institute of Technology (USA); W. Yueh, J. M. Roberts, Intel Corp. (USA); K. E. Gonsalves, The Univ. of North Carolina at Charlotte (USA)

$692313 \quad$ EUV resist development in Selete [6923-41]

D. Kawamura, K. Kaneyama, S. Kobayashi, J. J. S. Santillan, T. Itani, Semiconductor Leading Edge Technologies, Inc. (Japan)

692314 Photoresist induced contrast loss and its impact on EUV imaging extendibility [6923-42]

K. van Ingen Schenau, ASML (Netherlands); S. Hansen, B. Pierson, ASML (USA);

J. van Schoot, ASML (Netherlands) 
692315 Finite element modeling of PAG leaching and water uptake in immersion lithography resist materials [6923-43]

B. M. Rathsack, S. Scheer, Tokyo Electron America, Inc. (USA); Y. Kuwahara, J. Kitano, Tokyo Electron Kyushu Ltd. (Japan); R. Gronheid, C. Baerts, IMEC (Belgium)

692316 A new technique for studying photo-acid generator chemistry and physics in polymer films using on-wafer ellipsometry and acid-sensitive dyes [6923-44]

C.-T. Lee, Georgia Institute of Technology (USA); W. Yueh, J. M. Roberts, T. R. Younkin, Intel Corporation (USA); C. L. Henderson, Georgia Institute of Technology (USA)

692317 A comparison of the reaction-diffusion kinetics between model-EUV polymer and molecular-glass photoresists [6923-45]

S. Kang, K. Lavery, National Institute of Standards and Technology (USA); K.-W. Choi, National Institute of Standards and Technology (USA) and Intel Corp. (USA); V. M. Prabhu, W. Wu, E. K. Lin, National Institute of Standards and Technology (USA); A. De Silva, N. Felix, C. Ober, Cornell Univ. (USA)

692318 Lactones in $193 \mathrm{~nm}$ resists: What do they do? [6923-46]

H. Ito, H. D. Truong, P. J. Brock, IBM Almaden Research Ctr. (USA)

692319 Polymer matrix effects on acid generation [6923-47]

T. H. Fedynyshyn, R. B. Goodman, MIT Lincoln Lab. (USA); J. Roberts, Intel Corp. (USA)

$69231 \mathrm{~A}$ Acid-base equilibrium in chemically amplified resist [6923-48]

K. Natsuda, T. Kozawa, K. Okamoto, S. Tagawa, Osaka Univ. (Japan)

6923 IB Ionic photo-acid generators containing functionalized semifluorinated sulfonates for highresolution lithography [6923-49]

Y. Yi, R. Ayothi, C. K. Ober, Cornell Univ. (USA); W. Yueh, H. Cao, Intel Corp. (USA)

6923 1C Non-chemically amplified resists for $193 \mathrm{~nm}$ lithography [6923-50]

I. Nishimura, W. H. Heath, K. Matsumoto, W.-L. Jen, S. S. Lee, C. Neikirk, Univ. of Texas at Austin (USA); T. Shimokawa, K. Ito, JSR Corp. (Japan); K. Fujiwara, JSR Micro Inc. (USA);

C. G. Willson, Univ. of Texas at Austin (USA)

6923 ID Post-lithography characterization of ArF resists for $\mathbf{4 5} \mathbf{n m}$ node implant layers and beyond [6923-51]

A. Pikon, Rohm and Haas Electronics Materials (France); G. Pohlers, Rohm and Haas Electronics Materials (USA); S. Derrough, F. Milesi, J. Foucher, CEA/LETI/MINATEC (France)

6923 1E LWR reduction in low-k1 ArF-immersion lithography [6923-52]

K. Matsunaga, T. Oori, H. Kato, D. Kawamura, E. Shiobara, Toshiba Corp. (Japan);

Y. Inatomi, T. Kawasaki, M. Iwashita, Tokyo Electron Kyushu Ltd. (Japan); S. Ito, Toshiba Corp. (Japan)

6923 IF All-dry photoresist systems: physical vapor deposition of molecular glasses [6923-53] F. Pfeiffer, C. Neuber, H.-W. Schmidt, Univ. Bayreuth (Germany) 
692311 Water-developable negative-tone single-molecule resists: high-sensitivity nonchemically amplified resists [6923-57]

R. A. Lawson, C.-T. Lee, Georgia Institute of Technology (USA); W. Yueh, Intel Corp. (USA);

L. Tolbert, C. L. Henderson, Georgia Institute of Technology (USA)

$69231 \mathrm{~J}$ Adamantane-based molecular glass resist for 193-nm lithography and beyond [6923-58] S. Tanaka, N. Matsumoto, H. Ohno, Idemitsu Kosan Co., Ltd. (Japan); N. Hatakeyama, Idemitsu Chemicals U.S.A. Corp. (USA); K. Ito, K. Fukushima, Idemitsu Kosan Co., Ltd. (Japan); H. Oizumi, I. Nishiyama, ASET (Japan)

6923 1K Molecular glass photoresists based on acidolysis of acetal compounds [6923-59] L. Wang, X. Zhai, N. XU, Beijing Normal Univ. (China)

6923 IL Molecular glass resists for next generation lithography [6923-60]

A. De Silva, N. Felix, J. Sha, J.-K. Lee, C. K. Ober, Cornell Univ. (USA)

$69231 \mathrm{M}$ The effect of EUV molecular glass architecture on the bulk dispersion of a photo-acid generator [6923-61]

D. L. VanderHart, National Institute of Standards and Technology (USA); A. De Silva, N. Felix, Cornell Univ. (USA); V. M. Prabhu, National Institute of Standards and Technology (USA); C. K. Ober, Cornell Univ. (USA)

6923 IN Molecular resists for EUV and EB lithography [6923-62]

I. Takemoto, N. Ando, K. Edamatsu, Y. Lee, M. Takashima, H. Yokoyama, Sumitomo

Chemical Co., Ltd. (Japan)

\section{POSTERS: MATERIALS AND PROCESSES FOR IMMERSION LITHOGRAPHY}

692310 Options for high index fluids for third generation 193i lithography [6923-63]

S. Kruger, S. Revuru, S.-Z. Zhang, D. D. Vaughn II, E. Block, The Univ. at Albany (USA);

P. Zimmerman, Intel Assignee to SEMATECH (USA); R. L. Brainard, The Univ. at Albany (USA)

6923 IP High refractive-index resists composed of anionic photoacid generator (PAG) bound polymers for $193 \mathrm{~nm}$ immersion lithography [6923-64]

K. E. Gonsalves, M. Wang, N. S. Pujari, Univ. of North Carolina at Charlotte (USA)

6923 IR Application technology of stacked film with highly controlled edge structure [6923-67] K. Ichino, K. Tanouchi, T. Iseki, N. Ogata, T. Yamamoto, K. Yoshihara, A. Fujimoto, Tokyo Electron Kyushu Ltd. (Japan)

6923 iT The study of defect control and patterning performance for top coating free resist process [6923-69]

M.-S. Kim, H.-R. Jung, H.-W. Ryu, H.-G. Lee, S.-M. Hong, H.-J. Kim, S.-N. Park, M.-G. Gil, H.-S. Kang, Hynix Semiconductor Inc. (South Korea)

$69231 \mathrm{U} \quad$ Highly hydrophobic materials for ArF immersion lithography [6923-71]

Y. Takebe, N. Shirota, T. Sasaki, K. Murata, O. Yokokoji, Asahi Glass Co., Ltd. (Japan) 
$69231 \mathrm{~V}$ Improvements of adhesion and hydrophobicity of wafer bevel in water immersion lithography [6923-72]

T. Ishibash, Renesas Technology Corp. (Japan); M. Terai, Mitsubishi Electric Corp. (Japan); T. Hagiwara, Renesas Technology Corp. (Japan); T. Kumada, Mitsubishi Electric Corp. (Japan); T. Hanawa, Renesas Technology Corp. (Japan); Y. Takebe, O. Yokokoji, Asahi Glass Co., Ltd. (Japan); T. Fujiwara, H. Akiyama, Nikon Corp. (Japan)

6923 IW Process manufacturability evaluation for next generation immersion technology node [6923-73]

M. Enomoto, T. Shimoaoki, T. Otsuka, S. Hatakeyama, K. Nafus, R. Naito, Y. Terashita, T. Shibata, H. Kosugi, M. Jyousaka, Tokyo Electron Kyushu Ltd. (Japan); J. Mallmann, R. Maas, M. Blanco Mantecon, E. van Setten, J. Finders, S. Wang, C. Zoldesi, ASML Netherlands B.V. (Netherlands)

6923 1Y Formation mechanism of 193nm immersion defects and defect reduction strategies [6923-76]

Y. Wei, S. Brandl, F. Goodwin, Qimonda North America Corp. (USA)

$69231 \mathrm{~L}$ Synthesis of novel alpha-fluoroacrylates and related polymers for immersion lithography [6923-77]

T. Yamashita, T. Ishikawa, M. Morita, T. Kanemura, H. Aoyama, Daikin Industries, Ltd. (Japan)

692320 Characterization of resist and topcoat properties for immersion lithography [6923-78] K. Jain, Y. Hishiro, Micron Technology, Inc. (USA)

\section{Part Two}

POSTERS: MATERIALS AND PROCESSES FOR DOUBLE PATTERNING/DOUBLE EXPOSURE

$692321 \quad$ A lithographic and process assessment of photoresist stabilization for double-patterning using 172-nm photoresist curing [6923-79]

N. Bekiaris, H. Cervera, J. Dai, Sokudo Co., Ltd. (USA); R. Kim, Advanced Micro Devices, Inc. (USA); A. Acheta, Spansion LLC (USA); T. Wallow, J. Kye, H. J. Levinson, Advanced Micro Devices, Inc. (USA); T. Nowak, J. Yu, Applied Materials, Inc. (USA)

692322 Ion implantation as insoluble treatment for resist stacking process [6923-80]

H. Nakamura, T. Shibata, K. Rikimaru, S. Ito, S. Tanaka, S. Inove, Toshiba Corp. (Japan)

692323 Double patterning study with inverse lithography [6923-81]

S.-K. Kim, Hanyang Univ. (South Korea)

POSTERS: RESIST FUNDAMENTALS

692325 Photons, electrons, and acid yields in EUV photoresists: a progress report [6923-84]

R. Brainard, E. Hassanein, J. Li, Univ. at Albany (USA); P. Pathak, Univ. of Wisconsin, Madison (USA); B. Thiel, Univ. at Albany (USA); F. Cerrina, Univ. of Wisconsin, Madison (USA); R. Moore, M. Rodriguez, Univ. at Albany (USA); B. Yakshinskiy, E. Loginova, T. Madey, Rutgers Univ. (USA); R. Matyi, Univ. at Albany (USA); M. Malloy, A. Rudack, SEMATECH (USA); P. Naulleau, Univ. at Albany (USA); A. Wüest, K. Dean, SEMATECH (USA) 
692326 Ellipsometry studies of the kinetic of deprotection of thin $193 \mathrm{~nm}$ positive tone resist film [6923-85]

H. Ridaoui, LTM, CNRS (France); S. Derrough, C. Sourd, LETI, CER (France); H. Trouve,

A. Pikon, Rohm and Haas (France); J. H. Tortai, LTM, CNRS (France)

692327 Process-induced bias: a study of resist design and process implications [6923-86]

C. Fonseca, S. Scheer, M. Carcasi, Tokyo Electron America, Inc. (USA); T. Shibata, T. Otsuka, Tokyo Electron Kyushu Ltd. (Japan)

692328 ArF photoresist formulation influence on elliptical contact shape when using a dipole illumination [6923-87]

E. Tenaglia, STMicroelectronics (Italy); J. Ronsmans, JSR Micro N.V. (Belgium); D. De Simone, STMicroelectronics (Italy); T. Kimura, JSR Micro N.V. (Belgium); G. Cotti, STMicroelectronics (Italy)

692329 Dynamics of poly(4-hydroxystyrene) radical cation [6923-89]

K. Okamoto, T. Kozawa, S. Tagawa, Osaka Univ. (Japan)

6923 2A Dependence of acid generation efficiency on acid molecular structure and concentration of acid generator in chemically amplified EUV resist [6923-90]

R. Hirose, T. Kozawa, S. Tagawa, Osaka Univ. (Japan); T. Kai, T. Shimokawa, JSR Corp.

(Japan)

6923 2B Characterization of the latent image to developed image in model EUV photoresists [6923-91]

J. T. Woodward, National Institute of Standards and Technology (USA); K.-W. Choi, National Institute of Standards and Technology (USA) and Intel Corp. (USA); V. M. Prabhu, S. Kang, K. A. Lavery, W. WU, National Institute of Standards and Technology (USA); M. Leeson, Intel Corp. (USA); A. De Silva, N. M. Felix, C. K. Ober, Cornell University (USA)

6923 2C Study of de-protection reaction analysis system for EUV lithography [6923-92]

A. Sekiguchi, Y. Kono, Litho Tech Japan Corp. (Japan)

6923 2E RAFT technology for the production of advanced photoresist polymers [6923-96] M. T. Sheehan, DuPont Electronic Polymers L.P. (USA); W. B. Farnham, E.I. DuPont de Nemours (USA); H. Okazaki, J. R. Sounik, G. Clark, DuPont Electronic Polymers L.P. (USA)

6923 2F Effect of PAG and matrix structure on PAG acid generation behavior under UV and highenergy radiation exposure [6923-97]

C.-T. Lee, Georgia Institute of Technology (USA); M. Wang, K. E. Gonsalves, Univ. of North Carolina, Charlotte (USA); W. Yueh, J. M. Roberts, T. R. Younkin, Intel Corp. (USA);

C. L. Henderson, Georgia Institute of Technology (USA)

\section{POSTERS: ARCS AND MULTILAYER MATERIAL AND PROCESSES}

$69232 \mathrm{G}$ Plasma etch properties of organic BARCs [6923-98]

R. Huang, M. Weigand, Brewer Science, Inc. (USA)

$69232 \mathrm{H} \quad$ The design and evaluation of high barrier performance organic BARC material [6923-99] T. Ishida, R. Sakamoto, Y. Hiroi, Y. Sakaida, T. Hamada, Nissan Chemical Industries, Ltd. (Japan) 
$692321 \quad$ Development of high-performance tri-layer material [6923-100]

D. C. Owe-Yang, Shin-Etsu MicroSi (USA); T. Yano, T. Ueda, M. Iwabuchi, T. Ogihara, S. Shirai, Shin-Ełsu Chemical (Japan)

6923 2J High-etch-rate low-bias bow outgassing BARC via-filling materials for 193-nm ArF lithographic process [6923-101]

H. Yao, Z. Xiang, S. Mullen, J. Yin, W. Liu, J. Shan, E. Gonzalez, G. Lin, M. Neisser, AZ

Electronic Materials USA Corp. (USA)

6923 2K Advanced ultraviolet cross-link process and materials for global planarization [6923-102] S. Takei, Y. Horiguchi, T. Ohashi, B.-C. Ho, Y. Nakajima, Y. Mano, Nissan Chemical Industries, Ltd. (Japan); M. Muramatsu, M. Iwashita, Tokyo Electron Kyushu Ltd. (Japan); K. Tsuchiya, A. Samura, Y. Yamada, T. Yamaguchi, Tokyo Electron Ltd. (Japan)

$69232 \mathrm{~L}$ Gap-fill type HSQ/ZEP520A bilayer resist process-(I): HSQ-coated ZEP520A CD shrinkage for 32-nm trench patterns [6923-103]

W.-S. Chen, M.-J. Kao, M.-J. Tsai, Industrial Technology Research Institute (Taiwan, China)

$69232 \mathrm{M}$ Challenges of non-PFOS top antireflective coating material [6923-104]

S.-H. Hsu, Qimonda Dresden GmbH \& Co. OHG (Germany) and Nanya Technology Corp. (Taiwan, China); I. Vermeir, M. Scholze, M. Voigt, J. Gierth, A. Mittermeier, I. Mäge,

L. Voelkel, Qimonda Dresden GmbH \& Co. OHG (Germany)

$69232 \mathrm{~N} \quad$ KrF bilayer resist defects: cause, analysis, and reduction [6923-105]

B. Osborn, G. Quinto, Z. Zhang, C. Tang, S. Sakai, G. Nagatani, A. Minvielle, Spansion, Inc. (USA)

692320 Sub-45nm resist process using stacked-mask process [6923-106]

Y. Seino, K. Kobayashi, K. Sho, H. Kato, S. Miyoshi, K. Kikutani, J. Abe, H. Hayashi, T. Ohiwa,

Y. Oonishi, S. Ito, Toshiba Corp. (Japan)

$69232 \mathrm{P} \quad$ High-etch-rate bottom-antireflective coating and gap-fill materials using dextrin derivatives in via first dual-damascene lithography process [6923-107]

S. Takei, Y. Sakaida, T. Shinjo, K. Hashimoto, Y. Nakajima, Nissan Chemical Industries, Ltd. (Japan)

$69232 \mathrm{Q}$ Silicon-based anti-reflective spin-on hardmask materials for $\mathbf{4 5} \mathbf{~} \mathbf{m}$ pattern of immersion ArF lithography [6923-108]

S. K. Kim, H. M. Cho, S. R. Koh, M. Kim, H. C. Yoon, Y. Chung, J. S. Kim, T. Chang, Samsung Cheil Industries, Inc. (South Korea)

$69232 \mathrm{R}$ Optimization of BARC process for hyper-NA immersion lithography [6923-109]

K. Lee, J. Lee, S. Lee, D. Park, C. Bok, S. Moon, Hynix Semiconductor Inc. (South Korea)

6923 2T Development of new BARC for immersion process using hyper NA [6923-111]

H. J. Roh, M. H. Han, S. J. Kim, H. J. Kim, J. Kim, Dongjin Semichem Co. Ltd. (South Korea);

K. K. Kong, K. L. Lee, S. K. Lee, D. H. Park, Hynix Semiconductor Inc. (South Korea)

$69232 \mathrm{~V} \quad$ Novel spin-on organic hardmask with high plasma etch resistance [6923-114]

C.-I. Oh, J.-K. Lee, M.-S. Kim, K.-H. Yoon, H.-S. Cheon, N. Tokareva, J.-Y. Song, J.-S. Kim,

T.-W. Chang, Cheil Industries Inc. (South Korea) 
6923 2W Dual-layer dye-filled developer-soluble BARCs for 193-nm lithography [6923-115]

J. D. Meador, C. Beaman, C. Stroud, J. A. Lowes, Z. Zhu, D. J. Guerrero, R.-M. L. Mercado, Brewer Science, Inc. (USA); D. Drain, Missouri Univ. of Science \& Technology (USA)

$69232 \mathrm{X} \quad$ Low out-gassing organic spin-on hardmask [6923-116]

S. Minegishi, N. Yoshimura, M. Sato, Y. Konno, K. Konno, JSR Corp. (Japan); M. Slezak, JSR Micro (USA); J. Takahashi, S. Abe, Y. Yamaguchi, T. Shimokawa, JSR Corp. (Japan)

$69232 Y$ Gap-fill type HSQ/ZEP520A bilayer resist process-(II): HSQ island and spacer formation [6923-117]

W.-S. Chen, P.-Y. Gu, M.-J. Kao, M.-J. Tsai, Industrial Technology Research Institute (Taiwan, China)

$69232 Z$ Reflection control for immersion lithography at 45/32-nm nodes [6923-118] W.-J. Tseng, R.-H. Hsu, S. H. Hou, T.-H. Tseng, B. Lin, C. C. Yu, United Microelectronics Corp. (Taiwan, China); S. R. Kim, J. Y. Yu, G. Wayton, M. Ciambra, S. Coley, D. Praseuth, N. Pugliano, Rohm and Haas Electronic Materials (USA)

692330 Second-generation radiation sensitive 193-nm developable bottom antireflective coatings (DBARC): recent results [6923-119]

F. Houlihan, A. Dioses, L. Zhang, J. Oberlander, A. Krawicz, S. Vasanthan, M. Li, Y. Wei,

P. LU, M. Neisser, AZ Electronic Materials USA Corp. (USA)

$692331 \quad$ Effects of bake temperature and surface modifications on hardmask materials for trilayer applications [6923-120]

C. J. Neef, J. Finazzo, C. Nesbit, M. Weigand, Brewer Science, Inc. (USA)

\section{POSTERS: SIMULATION OF RESIST PROCESSES}

692332 A calibrated photoresist model for pattern prediction [6923-121]

Y. L. Hung, C. C. Liao, C.-L. Shih, Nanya Technology Corp. (Taiwan); J. J. Biafore,

S. A. Robertson, KLA-Tencor Corp. (USA)

\section{POSTERS: RESIST MATERIALS AND PROCESSES}

692333 Fabrication of 32-nm contact/via hole by photolithographic-friendly method [6923-122] T. Kawasaki, S. Shimura, F. Iwao, Tokyo Electron Kyushu Ltd. (Japan); E. Nishimura, M. Kushibiki, Tokyo Electron AT Ltd. (Japan); K. Hasebe, Tokyo Electron Tohoku Ltd. (Japan): M. Carcasi, M. Somervell, S. Scheer, Tokyo Electron America Ltd. (USA); H. Yaegashi, Tokyo Electron Ltd. (Japan)

692334 Evaluation of adamantane derivatives for chemically amplified resist: a comparison between ArF, EUV, and EB exposures [6923-123]

K. Furukawa, Mitsubishi Gas Chemical Co., Inc. (Japan); S. Seki, T. Kozawa, S. Tagawa, Osaka Univ. (Japan)

692335 Leading-edge adamantyl polymers designed for $193 \mathrm{~nm}$ lithography [6923-124] K. Fukushima, S. Tanaka, N. Matsumoto, H. Ohno, N. Kawano, H. Yamane, Idemitsu Kosan Co., Ltd. (Japan); N. Hatakeyama, Idemitsu Chemicals U.S.A. Corp. (USA); K. Ito, IdemitsU Kosan Co., Ltd. (Japan) 
692336 Wet trimming process for critical dimension reduction [6923-125]

S. X. Sun, B. A. Smith, A. Qin, Brewer Science, Inc. (USA)

692337 Impact of $\mathrm{HBr}$ and Ar cure plasma treatments on 193nm photoresists [6923-126] A. Bazin, STMicroelectronics (France); E. Pargon, X. Mellhaoui, Lab. des Technologies de la Microélectronique, CNRS (France); D. Perret, Rohm and Haas Electronic Materials (France); B. Mortini, STMicroelectronics (France); O. Joubert, Lab. des Technologies de la Microélectronique, CNRS (France)

692338 Molecular contamination control technologies for high volume production in high NA 193-nm lithography (phase II) [6923-127]

T. Nakano, T. Tanahashi, A. Imai, K. Yamana, T. Shimotsu, Nichias Corp. (Japan)

692339 Driving contact hole resolution to $45 \mathrm{~nm}$ using novel process enhancement techniques [6923-128]

W. Montgomery, S. Bennett, L. Huli, J. Weeks, A. Mackie, Univ. at Albany (USA)

6923 3A Resist reflow process for arbitrary $32 \mathrm{~nm}$ node pattern [6923-129]

J.-M. Park, I. An, H.-K. Oh, Hanyang Univ. (South Korea)

6923 3B Processing and modeling optimization for grayscale lithography [6923-131]

T. Dillon, M. Zablocki, J. Murakowski, D. Prather, Univ. of Delaware (USA)

6923 3C Advanced resist process enabling implementation of CD controllability for $32 \mathrm{~nm}$ and beyond [6923-132]

S. Shimura, F. Iwao, T. Kawasaki, Tokyo Electron Kyushu Ltd. (Japan); D. Shannon, TEL Technology Ctr., America LLC (Japan); Y. Tanaka, H. Yaegashi, Tokyo Electron Ltd. (Japan);

Y. Yamada, Tokyo Electron Kyushu Ltd. (Japan)

6923 3E Development of thick negative photoresists for electroplating applications [6923-134] C. Chen, R. Plass, E. Ng, S. Lee, S. Meyer, G. Pawlowski, AZ Electronic Materials USA Corp. (USA); R. Beica, Semitool, Inc. (USA)

6923 3F Synthesis and evaluation of novel resist monomers and copolymers for ArF lithography: Part II [6923-135]

O. Nakayama, T. Fukumoto, M. Tachibana, J. Sato, M. Kitayama, T. Kajiyashiki, Kuraray Co., Ltd. (Japan)

$69233 \mathrm{G}$ Line width roughness (LWR) performance of novel surface conditioner solutions for immersion lithography [6923-136]

B. J. Lu, E. T. Liu, A. Zeng, A. Tseng, S. Wu, B. Lin, C. C. Yu, United Microelectronics Corp. (Taiwan, China); L.-J. Meng, M. Jaramillo, Jr., M.-J. Liao, Air Products and Chemicals Inc. (USA)

$69233 \mathrm{H} \quad$ Effect of nanofiltration on photochemical integrity [6923-137]

H. Zhang, A. Wu, J. Wei, Entegris, Inc. (USA); R. Buschjost, Brewer Science (USA)

$692331 \quad$ Performance comparison of negative resists for copper rerouting and other electroplating applications [6923-138]

M. Toukhy, C. Chen, M. Paunescu, G. Pawlowski, AZ Electronic Materials USA Corp. (USA) 
6923 3J Post develop stain defect reduction [6923-139]

M. Harumoto, T. Kuroda, M. Sugiyama, A. Hisai, SOKUDO Co., Ltd. (Japan)

6923 3K Impact of surface treatment on resist reflow process [6923-141]

Y.-J. Yun, J.-H. Park, H. Choi, S. R. Park, K. Choi, J.-H. Kim, J.-W. Han, Dongbu HiTek (South Korea)

6923 3 Achieving small dimensions with an environmentally friendly solvent: photoresist development using supercritical $\mathrm{CO}_{2}$ [6923-142]

N. M. Felix, A. De Silva, J. Sha, C. K. Ober, Cornell Univ. (USA)

6923 3M Wafer shape compensation at the track PEB for improved CD uniformity [6923-143]

T. Michaelson, J. Dai, L. Chen, H. Cervera, B. Lue, H. Herchen, K. Vellore, N. Bekiaris, Sokudo USA (USA)

$69233 \mathrm{~N}$ Wafer warp caused by thick film resists acting as a permanent part of the device [6923-144]

R. Leuschner, M. Franosch, Infineon Technologies AG (Germany); T. Dow, Infineon Technologies AG (Austria)

692330 Characterization of array CD uniformity with respect to pattern density in $193 \mathrm{~nm}$ dry photolithography [6923-145]

V. Sipani, D. A. Kewley, K. Jain, E. R. Byers, B. Daybell, A. Krauth, Micron Technology, Inc. (USA)

$69233 Q \quad$ Non-ionic photoacid generators for chemically amplified resists: chromophore effect on resist performance [6923-147]

Y. Nishimae, H. Yamato, T. Asakura, M. Ohwa, Ciba Japan K. K. (Japan)

$69233 R$ The synthesis of novel ester acetal polymers and their application for chemically amplified positive i-line photoresist [6923-148]

L. Wang, Y. Huo, F. Kong, Beijing Normal Univ. (China)

692335 Study for aluminum metal patterning process with oxide hardmask in 90-nm s-flash memory device fabrication [6923-149]

S. I. Hwang, K. J. Yun, S. W. Ryu, K. H. Lee, J. W. Han, Dongbu Hitek (South Korea)

6923 3T Defect reduction using new digital valve dispensing technology [6923-150]

G. Standley, J. Kasson, Freescale Semiconductor (USA); B. Kidd, Integrated Designs, Inc. (USA)

$69233 \mathrm{U}$ Study for high voltage gate RIE process in LDI (LCD driver IC) device fabrication [6923-152] M. G. Lee, C. K. Jung, S. W. Ryu, K. H. Lee, J. W. Han, Dongbu Hitek (South Korea)

6923 3V Study of shallow trench isolation dry etching process using oxide hard mask and KrF photoresist in $90 \mathrm{~nm}$ stand-alone flash device [6923-153]

E. Cho, M. Lee, D. Shin, S. Hwang, S. Ryu, K. Lee, Dongbu HiTek (South Korea)

6923 3W 60-seconds puddle time: a tradition to overcome in CA resists: process optimization and defect elimination [6923-154]

E. Shalom, S. Zeid, Tower Semiconductor Ltd. (Israel) 
6923 3X Reflection control for line features of multiple pitches at hyper NA [6923-155]

M. Reilly, M. Wagner, Rohm and Haas Electronic Materials (USA); W. Montgomery, SUNY, CNSE (USA); N. Pugliano, Rohm and Haas Electronic Materials (USA); S. Robertson, KLA-

Tencor (USA)

$69233 \mathrm{Y}$ Improvement of 90nm technology VIA photo alignment through TiN hard mask removing above previous photo alignment mark area [6923-157]

W.-S. Liao, H.-H. Lin, Y.-H. Liu, M.-C. Tang, S.-Y. Huang, C.-H. Wu, United Microelectronics Corp. (Taiwan, China); Y.-G. Liaw, ASint Technology Corp. (Taiwan, China); T.-H. Chen, T. Shih, United Microelectronics Corp. (Taiwan, China); K.-M. Chen, National Nano Device Labs. (Taiwan, China)

$692341 \quad$ A comparison in dispense methodology for spray coat dispensing [6923-160] J. Coulter, Avago Technologies (USA); B. Kidd, Integrated Designs, L.P. (USA); J. Hawks, EV Group (USA)

POSTERS: EUV RESISTS

692342 EUV resist outgassing analysis in Selete [6923-161]

J. J. Santillan, S. Kobayashi, T. Itani, Semiconductor Leading Edge Technologies, Inc. (Japan)

$692344 \quad$ EUV resist based on low molecular weight PHS [6923-163]

M. Shirai, A. Kurosima, H. Okamura, Osaka Prefecture Univ. (Japan); K. Kaneyama, T. Itani, Semiconductor Leading Edge Technologies, Inc. (Japan)

692345 Quantitative analysis of EUV resist outgassing [6923-164]

S. Kobayashi, J. J. Santillan, T. Itani, Semiconductor Leading Edge Technologies, Inc. (Japan)

692346 Development of novel positive-tone resists for EUVL [6923-165]

T. Owada, Idemitsu Kosan Co., Ltd. (Japan); T. Watanabe, H. Kinoshita, Univ. of Hyogo (Japan); H. Oizumi, I. Nishiyama, Association of Super-Advanced Electronics Technologies (Japan)

692347 Development of partially fluorinated EUV-resist polymers for LER and sensitivity improvement [6923-166]

T. Sasaki, O. Yokokoji, Asahi Glass Co., Ltd. (Japan); T. Watanabe, H. Kinoshita, Univ. of Hyogo (Japan)

Author Index 
Downloaded From: https://www.spiedigitallibrary.org/conference-proceedings-of-spie on 26 Apr 2023

Terms of Use: https://www.spiedigitallibrary.org/terms-of-use 


\title{
Symposium Committees
}

\author{
Symposium Chair
}

Roxann L. Engelstad, University of Wisconsin, Madison (USA)

Symposium Cochair

Christopher J. Progler, Photronics, Inc. (USA)

Technical Organizing Committees

Executive Committee

Robert D. Allen, IBM Almaden Research Center (USA)

John A. Allgair, SEMATECH, Inc. (USA) and Advanced Micro

Devices, Inc. (USA)

Mircea Dusa, ASML US, Inc. (USA)

Roxann L. Engelstad, University of Wisconsin, Madison (USA)

Clifford L. Henderson, Georgia Institute of Technology (USA)

Bruno LaFontaine, Advanced Micro Devices, Inc. (USA)

Harry J. Levinson, Advanced Micro Devices, Inc. (USA)

Christopher J. Progler, Photronics, Inc. (USA)

Christopher J. Raymond, Nanometrics, Inc. (USA)

Michael L. Rieger, Synopsys, Inc. (USA)

Franklin M. Schellenberg, Mentor Graphics Corporation (USA)

Vivek K. Singh, Intel Corporation (USA)

Advisory Committee

Robert D. Allen, IBM Almaden Research Center (USA)

William H. Arnold, ASML (USA)

Timothy A. Brunner, IBM Thomas J. Watson Research Center (USA)

Ralph R. Dammel, AZ Electronic Materials USA Corporation (USA)

Roderick R. Kunz, MIT Lincoln Laboratory (USA)

Harry J. Levinson, Advanced Micro Devices, Inc. (USA)

Burn Lin, Taiwan Semiconductor Manufacturing Company, Ltd. (Taiwan)

Chris A. Mack, LithoGuru.com (USA)

Victor Pol, Freescale Semiconductor, Inc. (USA)

Michael T. Postek, National Institute of Standards and Technology (USA)

Luc Van den Hove, IMEC (Belgium)

C. Grant Willson, The University of Texas at Austin (USA)

Anthony Yen, Taiwan Semiconductor Manufacturing Company, Ltd. (Taiwan) and Cymer, Inc. (USA) 
Downloaded From: https://www.spiedigitallibrary.org/conference-proceedings-of-spie on 26 Apr 2023

Terms of Use: https://www.spiedigitallibrary.org/terms-of-use 


\title{
Conference Committee
}

\author{
Conference Chair
}

Clifford L. Henderson, Georgia Institute of Technology (USA)

Conference Cochair

Robert D. Allen, IBM Almaden Research Center (USA)

Program Committee

George G. Barclay, Rohm and Haas Electronic Materials (USA)

Sean D. Burns, IBM Thomas J. Watson Research Center (USA)

Ralph R. Dammel, AZ Electronic Materials USA Corporation (USA)

Douglas J. Guerrero, Brewer Science, Inc. (USA)

Christoph K. Hohle, Qimonda Dresden GmbH \& Company OHG

(Germany)

Qinghuang Lin, IBM Thomas J. Watson Research Center (USA)

Nobuyuki N. Matsuzawa, Sony Atsugi Technology Center (Japan)

Dah-Chung Owe-Yang, Shin-Ełsu MicroSi, Inc. (USA)

Adam R. Pawloski, Affymetrix, Inc. (USA)

Vivek M. Prabhu, National Institute of Standards and Technology (USA)

Ernisse S. Putna, Intel Corporation (USA)

Mark H. Somervell, Tokyo Electron America, Inc. (USA)

Gregory M. Wallraff, IBM Almaden Research Center (USA)

Session Chairs

1 Keynote Session

Robert D. Allen, IBM Almaden Research Center (USA)

2 Materials and Processes for Immersion Lithography I

Ralph R. Dammel, AZ Electronic Materials USA Corporation (USA)

Gregory M. Wallraff, IBM Almaden Research Center (USA)

3 Materials and Processes for Immersion Lithography II

George G. Barclay, Rohm and Haas Electronic Materials (USA)

Qinghuang Lin, IBM Thomas J. Watson Research Center (USA)

$4 \quad$ Materials and Processes for Double Patterning/Double Exposure

Robert D. Allen, IBM Almaden Research Center (USA)

Christoph K. Hohle, Qimonda Dresden GmbH \& Company OHG

(Germany) 
5 Molecular Resists

Clifford L. Henderson, Georgia Institute of Technology (USA)

Ralph R. Dammel, AZ Electronic Materials USA Corporation (USA)

6 Simulation of Resist Processes

Adam R. Pawloski, Affymetrix, Inc. (USA)

Robert D. Allen, IBM Almaden Research Center (USA)

$7 \quad$ ARCs and Multilayer Materials and Processes

Douglas J. Guerrero, Brewer Science, Inc. (USA)

Sean D. Burns, IBM Thomas J. Watson Research Center (USA)

8 EUV Resists

Ernisse S. Putna, Intel Corporation (USA)

Dah-Chung Owe-Yang, Shin-Ełsu MicroSi, Inc. (USA)

9 Resist Fundamentals

Clifford L. Henderson, Georgia Institute of Technology (USA)

Vivek M. Prabhu, National Institute of Standards and Technology (USA)

10 Resist Materials and Processes

Nobuyuki Matsuzawa, Sony Atsugi Technology Center (Japan)

Mark H. Somervell, Tokyo Electron America, Inc. (USA)

11 Joint Session on EUV Resists with Conference 6921

Ernisse S. Putna, Intel Corporation (USA)

Bruno M. Lafontaine, Advanced Micro Devices, Inc. (USA) 